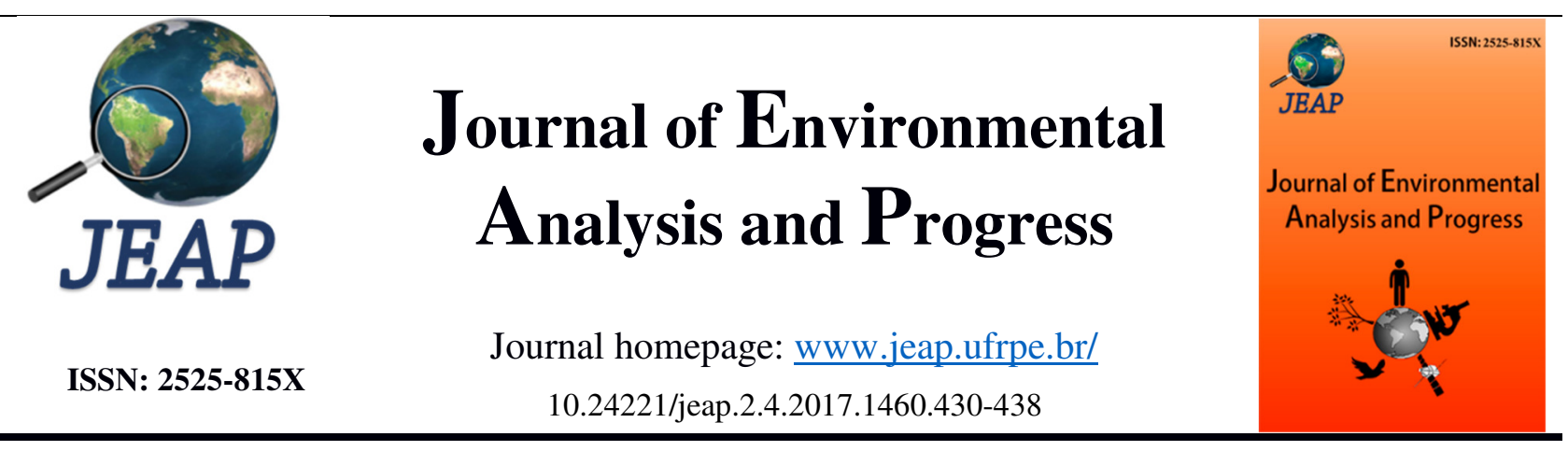

\title{
Avaliação das temperaturas mínimas e máximas para eventos de friagem no Pantanal
}

\section{Evaluation of minimum and maximum temperatures for Pantanal coldness events}

Müller Júnior Martins dos Santos ${ }^{\mathrm{a}}$, Luciana Barros Pinto ${ }^{\mathrm{a}}$, Gabriela Barbosa Irribarem ${ }^{\mathrm{a}}$, Lucas Fernando Carvalho da Conceição ${ }^{a}$, Carolina de Aguiar Monteiro ${ }^{a}$

a Universidade Federal de Pelotas-UFPel. Rua Gomes Carneiro, n. 1, Centro, Pelotas-RS, Brasil. CEP: 96010-610. Email: $\quad$ mllersantos@hotmail.com; $\quad$ luciana.pinto@ufpel.edu.br; $\quad$ g12irribarem@gmail.com; luucascarvalho93@gmail.com; carolina.am@outlook.com.

\begin{tabular}{l}
\hline A R T I C L E I N F O \\
\hline Recebido 30 Jun 2017 \\
Aceito 05 Set 2017 \\
Publicado 05 Out 2017
\end{tabular}

\begin{abstract}
A B S T R A C T
Despite the importance of the Pantanal, studies about coldness in the region are scarce in the literature. Several studies are geared to the Amazon region, showing less influence compared to the Pantanal. The study aimed to evaluate the phenomenon of coldness in the Pantanal, based on annual and monthly distribution of events, considering El Niño and the minimum and maximum temperatures in Coxim-MS. It was identified 75 events in 294 days under coldness conditions. Largest annual frequency occurred in 2009 and 2010, a total of 12 events for each year, concurring with the period of El Niño. The greatest monthly frequency was in August, totaling 16 events. It was reported two days with average temperature drop due to the cold and, later, raising the maximum temperature and the minimum temperature decrease continuity in the following days. The largest number of the annual frontal system does not imply in an increased number of cold days. July 2013 showed the lowest minimum temperature value in the period. Values with significant reductions in temperature, considering the normal climatological, did not show the lower minimum and maximum temperatures.
\end{abstract}

Keywords: Climatology, temperature deviation, seasonal distribution.

\section{R E S U M O}

Apesar da importância do Pantanal, estudos sobre friagens na região são escassos na literatura. Vários estudos são orientados para a região amazônica, mostrando menos influência em relação ao Pantanal. O estudo objetivou avaliar eventos de friagem no Pantanal, com base na distribuição anual e mensal dos eventos, considerando o El Niño e o comportamento das temperaturas mínimas e máximas, em Coxim-MS. Foram identificados 75 eventos em 294 dias sob efeito de friagem. A maior frequência anual ocorreu em 2009 e 2010, totalizando 12 eventos para cada ano, coincidindo com o período de El Niño ativo. A maior frequência mensal ocorreu em agosto, totalizando 16 eventos. Foi relatada uma média de dois dias, com queda de temperatura, em decorrência da friagem e, posteriormente, acréscimo na temperatura máxima e decréscimo na temperatura mínima nos dias seguintes. O maior número de sistema frontal anual não implicou em um número maior de dias frios. Julho de 2013 apresentou o menor valor de temperatura mínima no período. Valores com reduções significativas de temperatura, em relação à normal climatológica não apresentaram menores temperaturas mínimas e máximas.

Palavras-Chave: Climatologia, desvio de temperatura, distribuição sazonal. 


\section{Introdução}

O Pantanal é uma das principais áreas de preservação ambiental da atualidade (Farias \& Chan, 2006), está situado na região central da América do Sul, entremeio às áreas do Brasil, Paraguai e Bolívia. Em território brasileiro, o bioma do Pantanal possui uma área estimada em $150.355 \mathrm{~km}^{2}$ e está presente nos Estados de Mato Grosso do Sul e Mato Grosso (IBGE, 2004).

O clima do Pantanal apresenta uma estação chuvosa (nos meses de novembro a março) e outra seca (de abril a outubro) bem definidas (Garcia, 1986; Magalhães, 1992). Embora o clima da região pantaneira seja o tropical típico, ou Aw na classificação climática de Köppen (Garcia, 1986), com temperatura média anual em torno dos $25^{\circ} \mathrm{C}$ (Garcia, 1986), a atmosfera interage com diferentes massas de ar ocasionadas por sistemas transientes (Bjerknes, 1919), proporcionando em alguns casos temperatura mínima muito próximo de $0^{\circ} \mathrm{C}$ nos meses de outono e inverno (Nimer, 1989), quando há avanço de uma frente fria e na sua retaguarda tem-se a atuação de massa de ar frio oriundas do sul do continente para as baixas latitudes (Serra \& Ratisbonna, 1960).

A passagem de frentes-frias pelas regiões de latitudes tropicais, como a região do Pantanal e a amazônica gera alterações nas variáveis meteorológicas, é denominada por diversos autores como Friagem (Hamilton \& Tarifa, 1978; Fisch, 1996; Oliveira et al., 2004; entre outros). Entretanto, existe uma lacuna de estudos destas alterações para a região pantaneira, pois grande maioria dos estudos descrevem as características deste evento e sua influência na região amazônica.

Recentemente, Fernandes (2014) quantificou e analisou eventos de friagem na região do Pantanal entre o período de 2007 a 2012. Santos et al. (2016) buscaram analisar as temperaturas mínimas absolutas de um evento de friagem que atingiu a região de Coxim-MS, nordeste do Pantanal, em julho de 2013. Entretanto, uma melhor compreensão do fenômeno na região possibilita um aperfeiçoamento nas previsões destes eventos, amenizando possíveis perdas e transtornos causados pelos mesmos. Dentro deste contexto, o objetivo desse estudo é caracterizar a distribuição anual, mensal e diária de ocorrências de friagem na região do Pantanal no período de 2007 a 2014, avaliando as temperaturas mínimas e máximas da cidade de Coxim-MS.

\section{Material e Métodos}

Para a identificação dos eventos de friagem que ocorreram na região do Pantanal, Cuiabá-MG, no período de 2007 a 2014, foi utilizado o boletim mensal Climanálise, disponível no site do Centro de Previsão do Tempo e Pesquisas Climáticas do Instituto Nacional de Pesquisas Espaciais (CPTEC/INPE), seção de Sistemas Frontais e Frontogênese. Foram considerados como eventos de friagem os casos de Sistema Frontal (SF) que atingiram a cidade de Cuiabá, no Estado de Mato Grosso. Esta localidade foi tomada como base por estar localizada no nordeste do Pantanal e, uma vez que que as frentes frias no hemisfério Sul avançam no sentido Sudoeste-Nordeste, o sistema que chega até Cuiabá passa antes por toda a região do Pantanal.

Visando comparar os episódios históricos de EL Niño Oscilação Sul (ENOS) com as frequências dos eventos de friagens, foram utilizados os dados de ocorrência de El Niño/La Niña (1950-presente) disponiblizados pelo National Oceanic \& Atmospheric Administration (NOAA) (Tabela 1).

Tabela 1. Anos de ocorrência dos eventos de El Niño e La Niña durante o período de Estudo (2007 a 2014), onde os períodos quentes (vermelho) indicam presença de El Niño e os períodos frios (azul) indicam episódios de La Niña.

\begin{tabular}{ccccccccccccc}
\hline ANO & DJF & JFM & FMA & MAM & AMJ & MJJ & JJA & JAS & ASO & SON & OND & NDJ \\
\hline 2007 & 0.7 & 0.4 & 0.1 & -0.1 & -0.2 & -0.3 & -0.4 & -0.6 & -0.9 & -1.1 & -1.3 & -1.3 \\
2008 & -1.4 & -1.3 & -1.1 & -0.9 & -0.7 & -0.5 & -0.4 & -0.3 & -0.3 & -0.4 & -0.6 & -0.7 \\
2009 & -0.7 & -0.6 & -0.4 & -0.1 & 0.2 & 0.4 & 0.5 & 0.5 & 0.6 & 0.9 & 1.1 & 1.3 \\
2010 & 1.3 & 1.2 & 0.9 & 0.5 & 0.0 & -0.4 & -0.9 & -1.2 & -1.4 & -1.5 & -1.4 & -1.4 \\
2011 & -1.3 & -1.0 & -0.7 & -0.5 & -0.4 & -0.3 & -0.3 & -0.6 & -0.8 & -0.9 & -1.0 & -0.9 \\
2012 & -0.7 & -0.5 & -0.4 & -0.4 & -0.3 & -0.1 & 0.1 & 0.3 & 0.3 & 0.3 & 0.1 & -0.2 \\
2013 & -0.4 & -0.4 & -0.3 & -0.2 & -0.2 & -0.2 & -0.3 & -0.3 & -0.2 & -0.3 & -0.3 & -0.3 \\
2014 & -0.5 & -0.5 & -0.4 & -0.2 & -0.1 & 0.0 & -0.1 & 0.0 & 0.1 & 0.4 & 0.5 & 0.6 \\
\hline
\end{tabular}

A partir da identificação dos casos de friagem foram analisadas as temperaturas mínimas (Tmin, ${ }^{\circ} \mathrm{C}$ ) e máximas $\left(\mathrm{Tmax},{ }^{\circ} \mathrm{C}\right)$ diárias, com base nos dados da rede de estações meteorológicas automáticas do Instituto Nacional de Meteorologia
(INMET), disponíveis no Banco de Dados Meteorológicos para Ensino e Pesquisa (BDMEP) do INMET. Devido às falhas na série temporal dos dados das estações localizadas dentro do complexo pantaneiro, optou-se em utilizar apenas a estação 
com dados contínuos, resultando no uso exclusivo da estação da cidade de Coxim, localizada no nordeste do Pantanal (Figura 1).

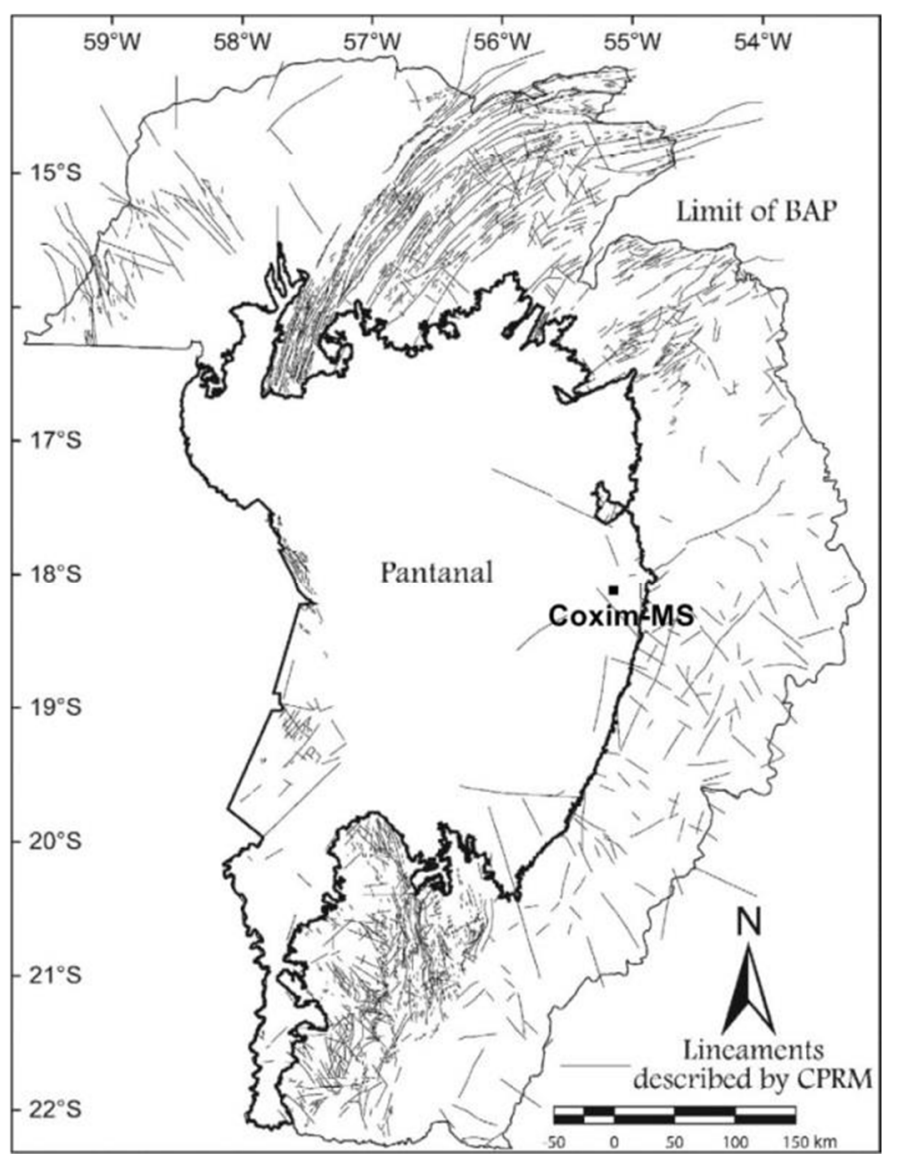

Figura 1. Localização geográfica do Pantanal e cidade de Coxim, em Cuiabá-MG. Fonte: Adaptado de Silva \& Abdon (1998).

Visando analisar o comportamento da temperatura, durante os casos de friagem, foram calculados os desvios em relação à Normal Climatológica (NC), do período de 1961 a 1990 (INMET), das temperaturas mínimas e máximas diária.

Para definir a duração de cada evento de friagem, foi considerado o dia da passagem do SF em Cuiabá como o primeiro dia do evento de friagem, e o último dia, como o dia no qual a Tmin ou Tmax deixaram de apresentam desvio negativo de temperatura em relação à $\mathrm{NC}$ ou o dia anterior em que ambas temperaturas (Tmin e Tmax) não apresentarem desvio negativo de temperatura, também em relação à NC. Após a seleção destes episódios, os mesmos foram organizados, contabilizados e avaliadas as distribuições anual, mensal e diária. Para a avalição diária de Tmin e Tmax foi considerado o D-1 ao dia anterior da friagem, D0 o primeiro dia, D+1 o segundo do evento, e assim sucessivamente.

\section{Resultados}

Durante o período de 2007 a 2014 foram identificados 75 eventos de friagens atuando sobre a região do Pantanal, tomando como critério de identificação os sistemas frontais que atingiram o município de Cuiabá-MT.

$\mathrm{Na}$ análise da distribuição anual dos eventos, verificou-se que a maior frequência de ocorrência de eventos de friagem foi nos anos de 2009 e 2010 , com um total de 12 eventos em cada ano (Figura 2). Entre os anos de 2009 e 2010, houve atuação do fenômeno atmosférico-oceânico caracterizado pelo aquecimento anormal das águas superficiais no oceano Pacífico Tropical, El Niño (Tabela 1), coincidindo com o período de maior frequência de eventos de friagens do estudo. De acordo com a Figura 2, o ano de 2014 foi o que apresentou menor frequência, registrando cinco eventos. 


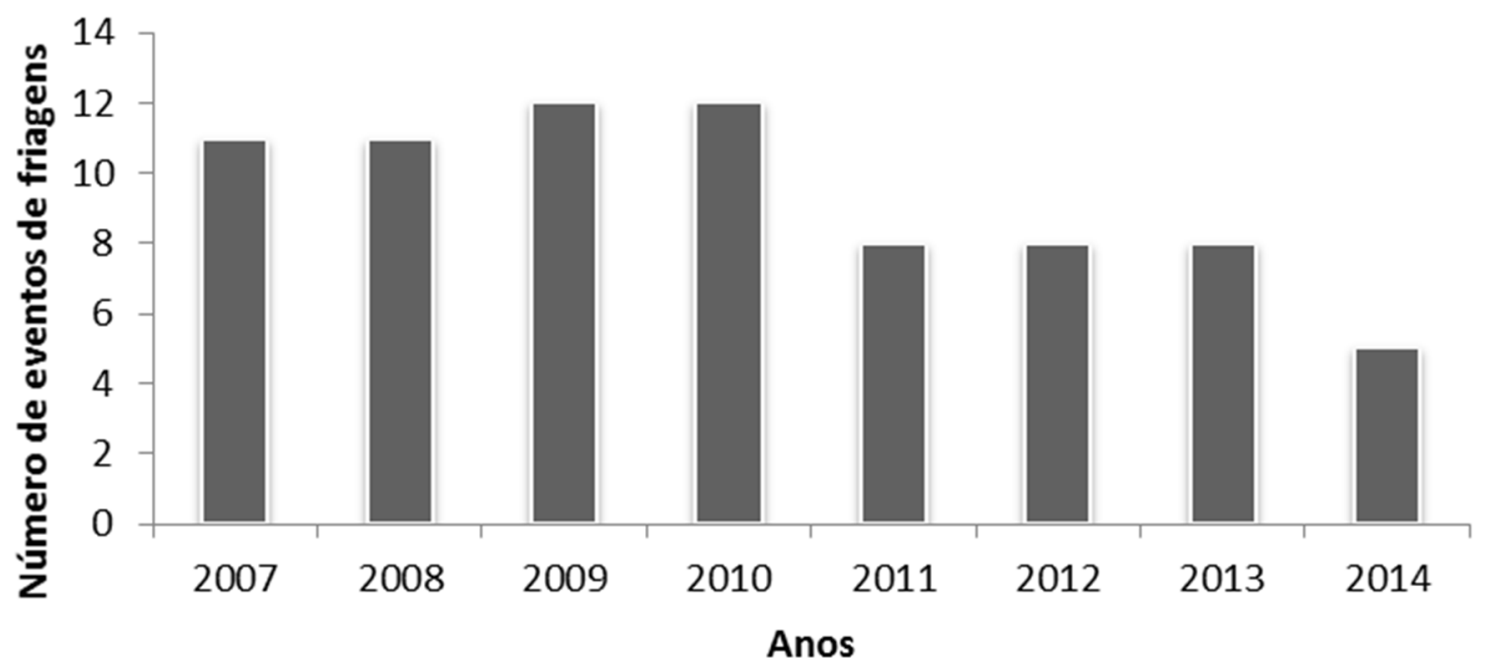

Figura 2. Distribuição anual dos eventos de friagens para a região do Pantanal, entre os anos de 2007 e 2014, com dados da cidade de Coxim-MS.

Considerando a distribuição mensal dos eventos de friagem (Figura 3), verificou-se que a maior frequência ocorreu em agosto, totalizando 16 eventos, seguido por maio, com 13 eventos, julho, com 12 eventos e junho e setembro, contabilizando
11 eventos em cada mês. Observou-se entre maio e setembro, meses mais frios na região, a ocorrência de maior frequência dos eventos, acumulando 63 friagens, correspondendo a $84 \%$ de todos os casos abordados neste estudo.

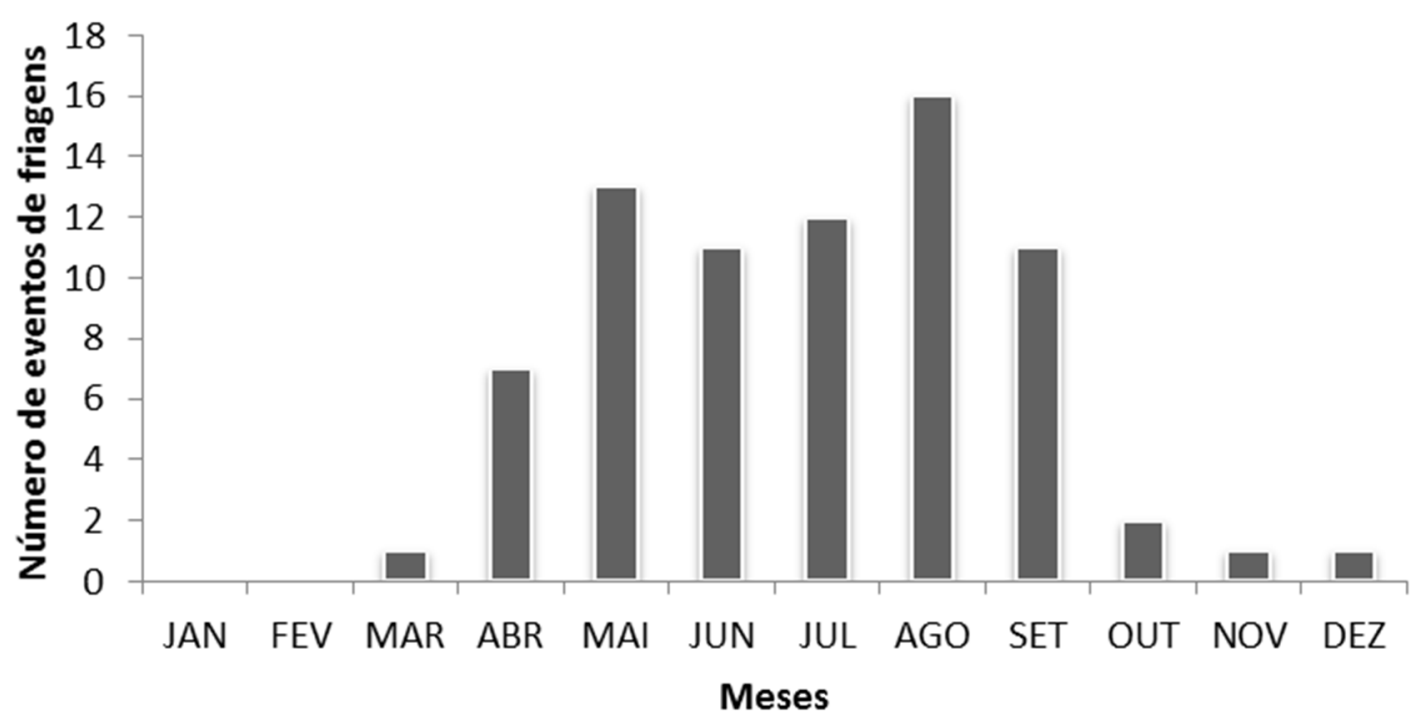

Figura 3. Distribuição mensal dos eventos de friagens para a região do Pantanal, entre os anos de 2007 e 2014 , com dados da cidade de Coxim-MS.

Considerando os 75 eventos observados nos oito anos de estudo e, um total de 294 dias sob o efeito de friagens, obteve-se uma média de 9,4 friagens.ano ${ }^{-1}$ e 36,8 dias.ano $^{-1}$ em Coxim. A duração média encontrada para cada evento de friagem foi de quatro dias.

Destacando o número de dias sob a influência do evento (Figura 4), o ano de maior frequência foi 2010, com um total de 50 dias.ano $^{-1}$.
Apesar do ano de 2009 ter apresentado o mesmo número de SF no período em relação a 2010, o número de dias ficou abaixo daqueles que ocorreram em 2007 (40 dias.ano-1), 2008 (39 dias.ano $\left.^{-1}\right)$ e $2012\left(38\right.$ dias.ano $\left.^{-1}\right)$ para a distribuição total de dias no ano, quando foram observados 37 dias.ano $^{-1}$. Esse resultado implica que, não necessariamente, um maior número de $\mathrm{SF}$ resultará em dias frios mais duradouros. 


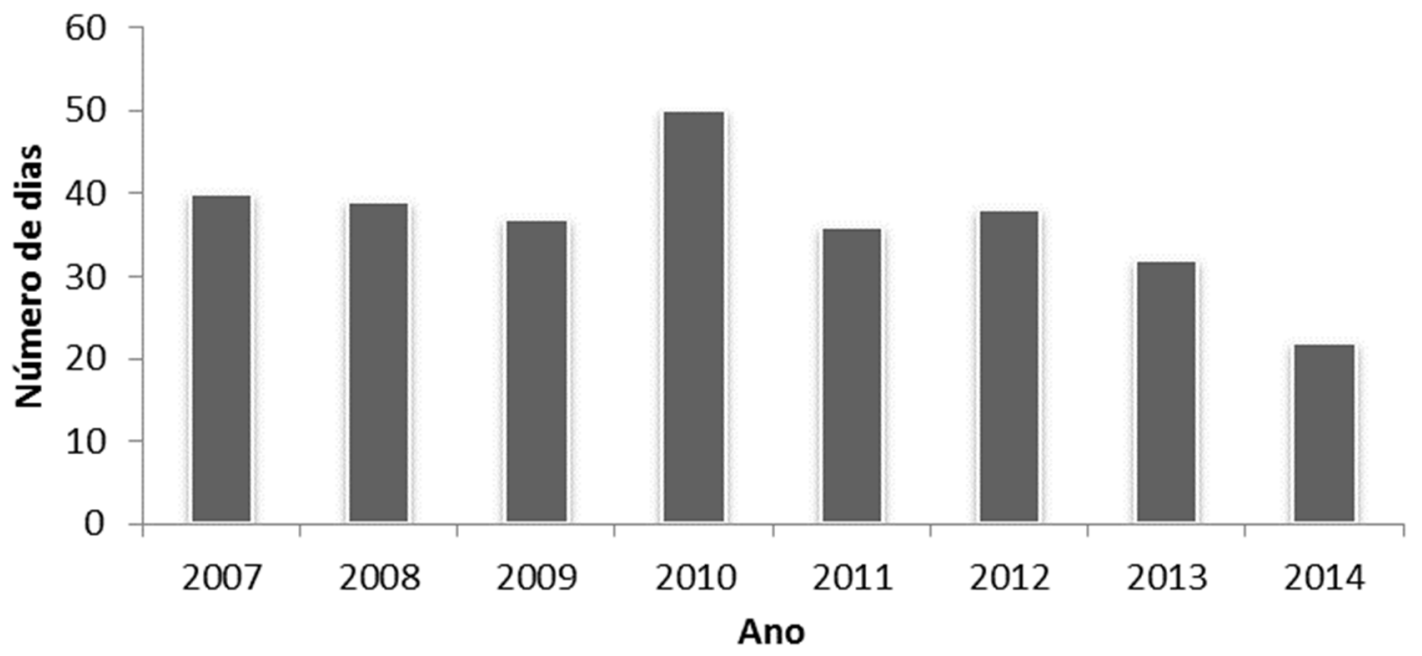

Figura 4. Número de dias do ano sob friagem em Coxim-MS (2007 a 2014).

Embora o mês de agosto tenha apresentando, durante todo o período, o maior número de $\mathrm{SF}$, os meses que apresentaram maior número de dias sob efeito de friagem ao longo de todos os anos do estudo foram maio e julho. $\mathrm{O}$ período estudado foi de 8 anos, ou seja, como maio e julho têm 31 dias cada, foram analisados 248 dias de maio e de julho nestes anos. Destes 248 dias, 60 dias (Figura 5) destes meses, no período total dos 8 anos, estiveram sob a atuação de friagem. Não obstante, o mês de agosto dos 248 dias dentro dos oito anos, totalizou 58 dias com friagem. Junho e setembro, dos 240 dias, apresentaram 43 e 33 dias, respectivamente, sob a influência de friagem (Figura 5), com 60 dias cada um (Figura 5). Não obstante, o mês de agosto totalizou 58 dias, seguido por junho e setembro, com 43 e 33 dias, respectivamente. Essa distribuição do número de dias mensalmente correspondeu a $86 \%$ de todo o período sob friagem. Ainda, analisando cada mês individualmente, os meses que mais apresentaram dias sob o fenômeno foram maio de 2010 e agosto de 2013, com 16 dias cada um.

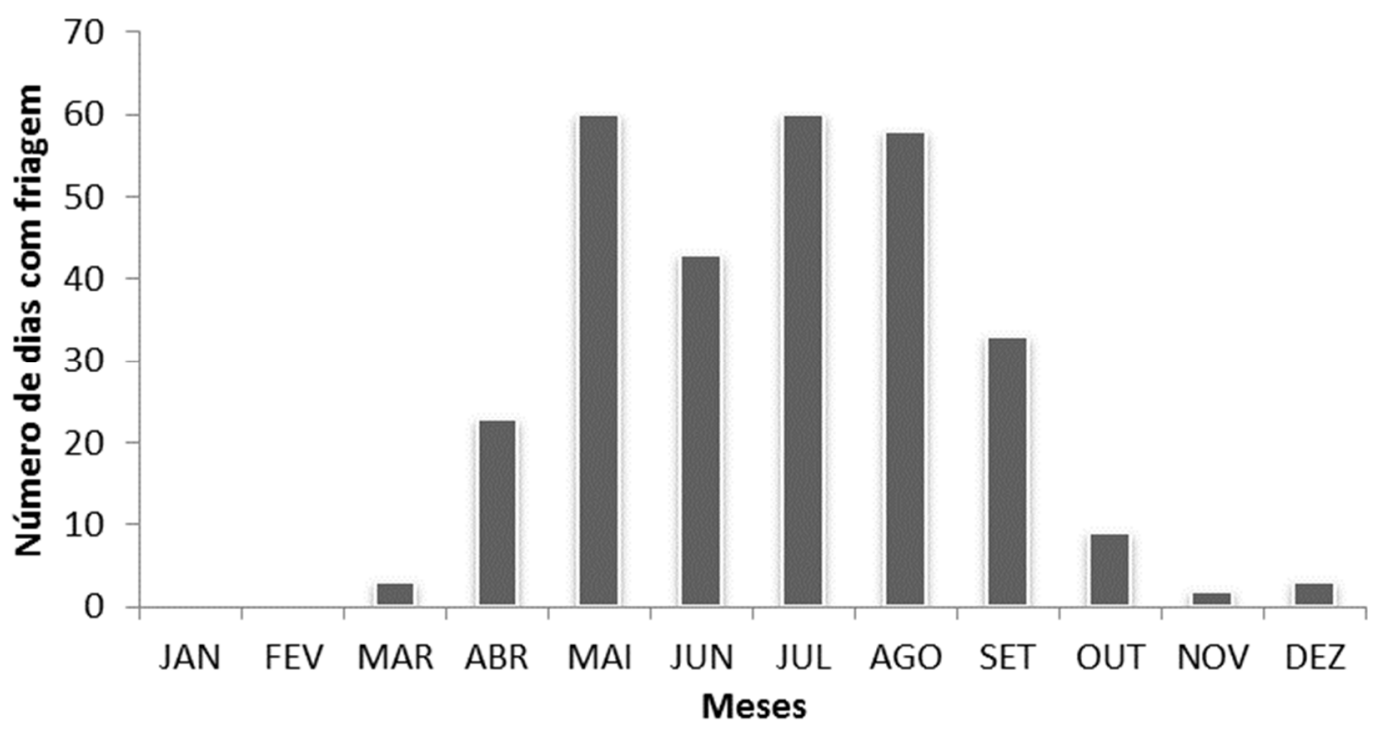

Figura 5. Distribuição mensal do número de dias sob efeito de eventos de friagens em Coxim-MS, entre os anos de 2007 e 2014.

A evolução temporal das Tmin e Tmax e a intensidade quanto ao desvio da $\mathrm{NC}$, a partir da média de cada dia dos 75 eventos de friagens, pode ser observada na Figura 6. Nota-se em D-1 Tmax elevada, que proporcionou a maior queda de temperatura de um dia para o outro, sob atuação da friagem, com decaimento de $5^{\circ} \mathrm{C}$ em D0. Logo, em
D0 a Tmin cai para $17,1^{\circ} \mathrm{C}$ e a $\operatorname{Tmax}$ para $26,6^{\circ} \mathrm{C}$, em média. Em $\mathrm{D}+1$, a Tmin cai para $14,2^{\circ} \mathrm{C}$ e a Tmax para $25,3^{\circ} \mathrm{C}$, menor valor de Tmax. A partir de $\mathrm{D}+2$ nota-se que a Tmax volta a ter acréscimo dos valores, subindo para $32^{\circ} \mathrm{C}$ em $\mathrm{D}+8$, enquanto que a Tmin continua suavemente com decréscimo, chegando aos $9,3^{\circ} \mathrm{C}$ em $\mathrm{D}+8$. 


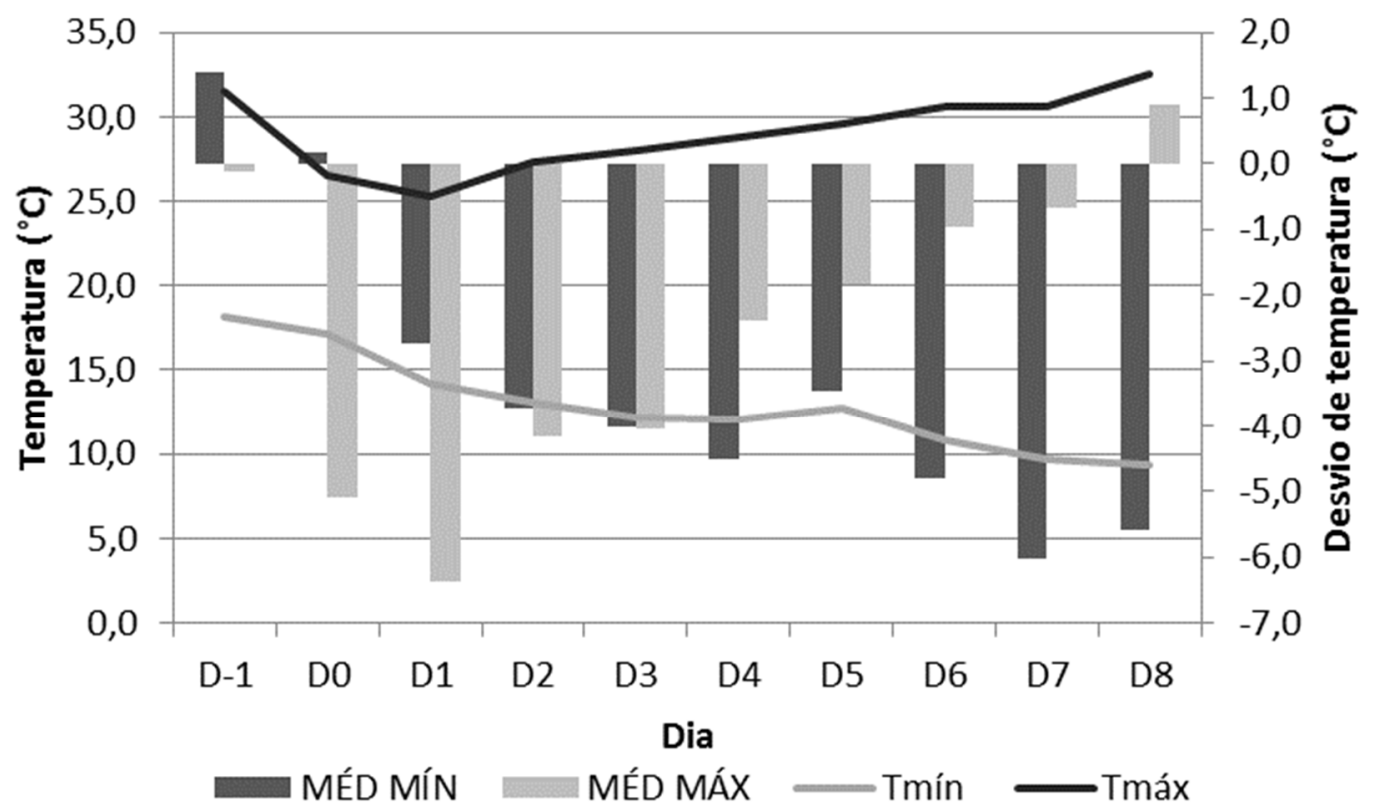

Figura 6. Evolução diária das médias de temperaturas mínimas (Tmin), máximas (Tmax) e desvio de temperatura $\left({ }^{\circ} \mathrm{C}\right)$ em relação à normal climatológica em eventos de friagem para a cidade de Coxim-MS, no período de 2007 a 2014.

Eventos de friagens apresentaram os maiores Desvio de temperatura mínima (Dtmin) e Desvio de temperatura máxima (Dtmax) em seus respectivos meses e anos de ocorrência (Tabela 2). Essa anomalia foi avaliada reduzindo o valor, tanto da Tmin como da Tmax, no dia do evento da média de cada mês para o respectivo SF. Considerando o maior valor de Dtmin $\left(-12,5^{\circ} \mathrm{C}\right)$ de todo o período em Coxim, o mês de julho de 2013 também mostrou a menor Tmin $\left(2,4^{\circ} \mathrm{C}\right)$. O maior Dtmax ($16,4^{\circ} \mathrm{C}$ ) ocorreu em julho de 2013 e 2014 , com Tmax de apenas $15,2^{\circ} \mathrm{C}$.

Tabela 2. Desvio negativo da Tmin e Tmax dos eventos de friagem em Coxim-MS, no período de 2007 a 2014.

\begin{tabular}{lcccccc}
\hline Mês & Tmin & Tmax & NC Tmin & NC Tmax & Dtmin & Dtmax \\
& & & & $(\mathbf{C})$ & $\ldots . .$. \\
\hline Março & 15,0 & 26,7 & 21,7 & 32,3 & $-6,7(2012)$ & $-5,6(2012)$ \\
Abril & 10,3 & 22,2 & 20,3 & 32,0 & $-10,3(2012)$ & $-9,8(2008)$ \\
Maio & 5,5 & 18,2 & 17,4 & 30,7 & $-11,9(2010)$ & $-12,5(2008)$ \\
Junho & 5,8 & 17,5 & 14,9 & 29,5 & $-9,1(2009)$ & $-12.0(2011)$ \\
Julho & 2,4 & 15,2 & 14,9 & 31,6 & $-12,5(2013)$ & $-16,4(2013 / 14)$ \\
Agosto & 5,4 & 18,0 & 15,5 & 32,5 & $-10,1(2013)$ & $-14,5(2013)$ \\
Setembro & 7,6 & 23,3 & 18,7 & 32,7 & $-11,1(2008)$ & $-9,4(2009)$ \\
Outubro & 18,9 & 22,6 & 20,8 & 33,7 & $-1,9(2010)$ & $-11,1(2008)$ \\
Novembro & 19,7 & 27,9 & 21,6 & 33,0 & $-1,9(2010)$ & $-5,1(2010)$ \\
Dezembro & 19,8 & 27,1 & 22,1 & 32,0 & $-2,3(2010)$ & $-4,9(2010)$ \\
\hline
\end{tabular}

\section{Discussão}

Tomando como critério de identificação os sistemas frontais que atingiram o município de Cuiabá-MT, Fernandes (2014) identificou 60 casos de friagens entre 2007 e 2012, mesmo valor encontrado neste estudo para o período de 2007 a 2012.

Entre os anos de 2009 e 2010, houve atuação do fenômeno atmosférico oceânico caracterizado pelo aquecimento anormal das águas superficiais no oceano Pacífico Tropical, El Niño (Tabela 1), coincidindo com o período de maior frequência de eventos de friagens do estudo. Esse comportamento foi explicado por Fedorova \& Carvalho (2000), onde mostraram que há uma maior frequência de frentes frias dentro da faixa de latitudes $20^{\circ} \mathrm{S}$ e $40^{\circ} \mathrm{S}$ com atuação dos fenômenos de El Niño do que La Niña. Apesar da diferença latitudinal enunciada pelos autores, essas frentes frias podem deslocar-se para as latitudes baixas, como a região do Pantanal.

Considerando a distribuição mensal dos eventos de friagem, os resultados encontrados concordam com Gan \& Rao (1991), quando 
elaboraram uma climatologia das ciclogêneses no sul da América do Sul, de janeiro de 1979 a dezembro de 1988, onde constaram o mês de maio com maior frequência, uma vez que a ciclogênese é um mecanismo que impulsiona as massas de ar frio das altas latitudes para as baixas latitudes (Seluchi, 2009; Garreaud, 2000), e destacaram que o período de menor frequência ocorreu durante o verão (dezembro a fevereiro). Garreaud (2000) encontrou resultado similar para a região Amazônica entre os anos de 1979 e 1995, onde o período de abril a setembro foi o de maior frequência; corroborando o estudo de Fernandes (2014), que identificou maio e agosto com maiores frequências de eventos de friagem.

Os resultados encontrados, em relação ao número de eventos de friagem por ano $(9,4$ friagens.ano $\left.{ }^{-1}\right)$ e número de dias médios sob atuação por ano (36,8 dias.ano $\left.{ }^{-1}\right)$, também foram analisados por outros autores, como Brinkman et al. (1971), Brinkman \& Ribeiro (1972), Fisch (1996), Cavalcanti \& Kousky (2009) e Fernandes (2014). Os três primeiros estudos foram aplicados para a região amazônica. Brinkman et al. (1971) e Brinkman \& Ribeiro (1972) identificaram, na Amazônia Central, a ocorrência de duas a três friagens por ano, durante os meses de seca (junho a outubro). Fisch (1996), aplicando uma estatística simples do número de eventos de friagem na região do Sul do Pará, durante os anos de 1992 e 1993, encontrou uma frequência de ocorrência de sete casos por ano, durante os meses de maio a agosto. Cavalcanti \& Kousky (2009) encontraram resultado superior ao observado neste estudo, com intervalo de 15 a 20 friagens.ano ${ }^{-1}$ para a região do Pantanal, no período de 1979 a 2005. Fernandes (2014) encontrou valor um pouco superior de 10 friagens.ano ${ }^{-1}$, principalmente quanto ao critério de identificação de friagem, e superior, em número de dias, com 44,8 dias.ano ${ }^{-1}$.

Destacando o número de dias sob influência do evento, os resultados implicaram que, não necessariamente, um maior número de $\mathrm{SF}$ resultará em dias frios mais duradouros.

A duração média encontrada para cada evento de friagem nesse estudo está de acordo com o estimado na literatura, entre dois a cinco dias para a Amazônia (Nimer, 1989; Pinto Jr.; Silva, 2012). Marengo et al. (1997) observaram que o tempo para que as variáveis meteorológicas voltem às condições normais, durante um evento de friagem, varia, em média, entre cinco e seis dias.

A evolução temporal das Tmin, Tmax e a intensidade quanto ao desvio da $\mathrm{NC}$, a partir da média de cada dia dos 75 eventos de friagens, corrobora com o estudo de Pinto Jr. e Silva (2012), onde relataram uma média de dois dias com queda nas temperaturas em decorrência da friagem. $\mathrm{O}$ comportamento da Tmin deve-se ao padrão típico de circulação em superfície, onde o anticiclone pós-frontal mantém características continentais o que favorece intensificar a perda radiativa durante a noite e em consequência provocar temperaturas mínimas mais baixas (Escobar, 2007). Pode-se observar que, não necessariamente, valores com reduções significativas de temperatura apresentaram menores Tmin e Tmax, como o ocorrido em abril e junho.

\section{Conclusão}

Um maior número de $\mathrm{SF}$ anual não implicou em maiores dias frios no respectivo ano. Valores com reduções significativas de temperatura, não necessariamente, apresentaram menores Tmin e Tmax.

$\mathrm{O}$ estudo dessa região mostrou ser de grande interesse para uma melhor compreensão da influência do fenômeno de friagem na modificação da vida da população durante sua ocorrência, assim como a regularidade das condições ambientais da região.

\section{Agradecimentos}

Os autores agradecem ao Conselho Nacional de Desenvolvimento Científico e Tecnológico $(\mathrm{CNPq})$ pelo apoio financeiro ao projeto de pesquisa.

\section{Referências}

AMORIM NETO, A. C. 2013. Estrutura e evolução de frentes frias intensas na região amazônica brasileira. Tese (Doutorado) Instituto Nacional de Pesquisas da Amazônia, Manaus. 182p.

BJERKNES, J. 1919. On the structure of moving cyclones. In: Monthly Weather Review, v. 47, n. 2, p. 95-99.

BRINKMANN, W. L.; WEINMAN, J. A.; GOES RIBEIRO, M. N. 1971. Air temperature in central Amazonia I. In: Acta Amazonica, v. 1, n. 1, p. 5156.

BRINKMANN, W. L.; GOES RIBEIRO, M. N. 1972. Air temperature in central Amazonia III. In: Acta Amazonica, v. 2, n. 3, p. 27-32.

CAVALCANTI, I. F. A.; KOUSKY, V. E. 2009. Frentes frias sobre o Brasil. In: CAVALCANTI, I. F. A; FERREIRA, N. J.; SILVA, M. G. A. J.; DIAS, M. A. F. S. (org.) Tempo e Clima no Brasil. 1ed. São Paulo: Oficina de Textos. v. 1, pp. 133147. 
CLIMANÁLISE. Boletim Climanálise. Disponível em:

http://climanalise.cptec.inpe.br/ rclimanl/boletim. Acesso em: 10 mai. 2016.

ESCOBAR, G. C. J. 2007. Padrões sinóticos associados a ondas de frio na cidade de São Paulo. Revista Brasileira de Meteorologia, v. 22, n. 2, p. 241-254.

FARIAS, S. E. M.; CHAN, C. S. 2006. Simulação das características micrometeorológicas para o Pantanal sul-mato-grossense. In: Simpósio de Geotecnologias no Pantanal, 1. 2006, Campo Grande-MS. Anais do.... Embrapa Informática Agropecuária/INPE, pp. 57-66.

FEDOROVA, N.; CARVALHO, M. H. 2000. Processos sinóticos em anos de La Niña e de El Niño. Parte 11: Zonas frontais. Revista Brasileira de Meteorologia, v. 15, n. 2, p. 57-72.

FERNANDES, B. M. 2014. Estudo de friagem na região do Pantanal. Trabalho de Conclusão de Curso de Graduação, Universidade Federal de Pelotas. Pelotas, Rio Grande do Sul, Brasil. 66p.

FISCH, G. F. 1996. Camada limite amazônica: aspectos observacionais e de modelagem. Tese de Doutorado, Instituto Nacional de Pesquisas Espaciais. São José dos Campos, São Paulo, Brasil. $180 \mathrm{p}$.

FORTUNE, M. A.; KOUSKY, V. E. 1983: “Two severe freezes in Brazil: Precursors and synoptic evolution", M.W.R., v. 109, p. 599-610.

GAN, M. A.; RAO, V. B. 1991: Surface Cyclogenesis over South America, Mon. Weath. Rev., v. 119, p. 1293-1302.

GARCIA, E. A. C. 1986. Estudo técnicoeconômico da pecuária bovina de corte do Pantanal Mato-Grossense. Embrapa-CPAP. Documentos, 4. Corumbá, Mato Grosso do Sul, Brasil. 150p.

GARREAUD, R. D. 2000. Cold air incursions over subtropical South America: mean structure and dynamics. Monthly Weather Review, v. 128, n. 7, p. 2544-2559.

GRADELLA, F. S. 2008. Aspectos da dinâmica hidroclimática da Lagoa Salina do Meio na fazendo Nhumirim e seu entorno, Pantanal da Nhecolândia, MS, Brasil. Dissertação de Mestrado, Universidade Federal do Mato Grosso do Sul. Aquidauana, Mato Grosso do Sul, Brasil. 75p.
HAMILTON, M. G.; TARIFA, J. R. 1978. Synoptic aspects of a polar outbreak leading to frost in tropical Brazil, July, 1972. Monthly Weather Review, v. 106, n. 11, p. 1545-1556.

IBGE. Instituto Brasileiro de Geografia e Estatística. Mapa de Biomas e de Vegetação. Disponível em: http://cidades.ibge.gov.br/xtras/home.php. Acesso em: 05 abr. 2004.

INMET. Instituto Nacional de Meteorologia. Disponível em: http://www.inmet.gov.br/projetos/rede/pesquisa/. Acesso em: 04 jan. 2016.

LONGO, M.; CAMARGO, R.; SILVA DIAS, M. A. F. 2004. Análise das Características Dinâmica e Sinóticas de um Evento de Friagem Durante a Estação Chuvosa no Sudoeste da Amazônia. Revista Brasileira de Meteorologia, v. 19, n. 1, p. 59-72.

MAGALHÃES, N. W. 1992. Conheça o Pantanal. São Paulo: Terragraph, 390p.

MARENGO, J. A.; NOBRE, C.; CULF, A. D. 1997. Climatic impacts of "friagens" in forested and deforested areas of the Amazon basin. Journal of Applied Meteorology, v. 36, n. 11, p. 15531566.

NIMER, E. 1989. Geografia do Brasil-Região Centro-Oeste. Rio de Janeiro, IBGE, v. 1, p. 23-34.

OLIVEIRA, P. J.; ROCHA, E. J. P.; FISCH, G.; KRUIJT, B.; RIBEIRO, J. B. M. 2004. Efeitos de um evento de friagem nas condições meteorológicas na Amazônia: um estudo de caso. Acta Amazonica, v. 34, n. 4, p. 613-619.

PINTO JR., S. C.; SILVA, C. A. 2012. A dinâmica climática do Mato Grosso do Sul e as queimadas do ano de 2009: uma análise têmporo-espacial a partir das Imagens do NOAA-15. Acta Geográfica, v. Ed. Especial, pp. 223-237.

RODELA, L. G. 2006. Unidades de vegetação e pastagens nativas do Pantanal da Nhecolândia, Mato Grosso do Sul. Tese de Doutorado, Universidade de São Paulo. São Paulo, São Paulo, Brasil. 222p.

SANTOS, M. J. M.; CONCEICAO, L. F. C.; MONTEIRO, C. A.; PINTO, L. B. 2016. Fenômeno intenso de friagem em julho de $2013 \mathrm{em}$ Coxim-MS, norte do Pantanal: análise do ambiente 
sinótico. XXV Congresso de Iniciação Científica. Universidade Federal de Pelotas. Pelotas, Rio Grande do Sul, Brasil.

SATYAMURTY, P.; FONSECA, J. F. B.; BOTINO, M. J.; SELUCHI, M. E.; LOURENÇO, M. C. M.; GONÇALVES, L. G. 2002. An early freeze in southern Brazil in April 1999 and inst NWP guidance. Meteorol. Appl., v. 9, n. 1, p. 113128.

SELUCHI, M. E. 2009. Geadas e Friagens. In: CAVALCANTI, I. F. A; FERREIRA, N. J.;
SILVA, M. G. A. J.; DIAS, M. A. F. S. (org.) Tempo e Clima no Brasil. 1 ed. São Paulo: Oficina de Textos. v. 1, p. 149-167.

SERRA, A.; RATISBONNA, L. 1960. As massas de ar da América do Sul: $1^{\text {a }}$ e $2^{\text {a }}$ partes. Revista Geográfica, Rio de Janeiro, Instituto Panamericano de Geografia e História, v. 51-52, p. 1959-1960.

SILVA J. S. V.; ABDON, M. M. 1998. Delimitação do Pantanal brasileiro e suas subregiões. EMBRAPA, v. 33 (Especial), p. 17031711. 